\title{
Low-Complexity Erasure Insertion in Frequency-Hopping Spread-Spectrum Communications Subjected to Fading and Partial-Band Interference
}

\author{
Lie-Liang Yang and Lajos Hanzo \\ Dept. of ECS, University of Southampton, SO17 1BJ, UK. \\ Tel: +44-703-593 125, Fax: +44-703-594 508 \\ Email: llydecs.soton.ac.uk and lh@ecs.soton.ac.uk \\ http: //www-mobile.ecs.soton.ac.uk
}

\begin{abstract}
In this paper we propose two novel low-complexity, low-delay erasure insertion schemes, namely the Output Threshold Test (OTT) and joint Maximum Output and Ratio Threshold Test (MO-RTT). The employment of the OTT and MO-RTT is beneficial in the context of the 'Errors-And-Erasures (EAE)' Reed-Solomon (RS) decoding in a Slow Frequency-Hopping based Spread-Spectrum (SFH/SS) system using $M$-ary Frequency-Shift Keying (MFSK). The statistics of the erasure insertion related decision variables associated with the OTT, MO-RTT as well as with the Ratio Threshold Test (RTT) are investigated, when the channel of each FH slot is modeled as flat Nakagami- $m$ fading. The transmitted signals also experience both Additive White Gaussian Noise (AWGN) as well as Partial-Band Gaussian Interference (PBGI). The performance of the proposed erasure insertion schemes and that of the erasure insertion scheme using the RTT is investigated and compared in the context of RS coded SFH/SS systems using MFSK modulation.
\end{abstract}

\section{INTRODUCTION}

In Slow Frequency-Hopping Spread-Spectrum (SFH/SS) wireless systems Forward Error-Correction (FEC) codes, such as for example Reed-Solomon (RS) codes are often used, in order to mitigate the performance degradation due to background noise, intentional and unintentional interference as well as due to channel fading $[1,2]$. In the context of RS coded SFH/SS systems using MFSK modulation, 'Errors-And-Erasures (EAE)' decoding is preferable to 'ErrorCorrection-Only (ECO)' decoding, since EAE decoding can typically achieve a significantly lower codeword decoding error probability, than ECO decoding, provided that a reliable erasure insertion scheme is invoked. In recent years the performance of RS coded SFH/SS systems using EAE decoding based on erasure insertion schemes, such as the Ratio Threshold Test (RTT) [3] or the Bayesian approach [4], has received wide attention.

In this contribution two novel erasure insertion schemes, namely the Output Threshold Test (OTT) and joint Maximum Output and Ratio Threshold Test (MO-RTT), which have similar complexity to the classic RTT [3] are proposed for employment in RS coded SFH/SS systems using MFSK modulation. Their performance as well as the performance of the RTT is investigated, when the channel is modeled

This work has been funded in the framework of the IST project IST-199912070 TRUST, which is partly funded by the European Union. The authors would like to acknowledge the contributions of their colleagues. as a flat Nakagami- $m$ fading medium [5] and the transmitted signals are also exposed to Patial Band Gaussian Interference (PBGI) $[1,2]$. We derive the exact Probability Density Functions (PDF) of the decision variables used for making erasure insertion decisions associated with the OTT, RTT and the MO-RTT erasure insertion schemes over flat Nakagami- $m$ fading channels, under both the hypotheses that the $M$-ary demodulated symbol is correct $\left(H_{1}\right)$ and incorrect $\left(H_{0}\right)$. These PDFs are then used for investigating the characteristics of the OTT, RTT as well as MO-RTT over the above-mentioned Nakagami$m$ fading channels. The performance of the RS coded SFH/SS system using MFSK modulation assisted by EAE decoding employing the OTT, RTT and MO-RTT is estimated and also compared with each other.

\section{SYSTEM OVERVIEW}

The system under consideration was described in $[1,2]$, and the reader might like to consult these references for their description. In the system considered SFH/SS using MFSK modulation, noncoherent demodulation based on square-law detection, and an extended $\operatorname{RS}(N, K)$ code over $\mathrm{GF}\left(2^{b}\right)$ are employed. We assume that $M=N=2^{b} \geq 4$, so that each $b$-bit RS code symbol describes an $M$-ary MFSK symbol. With the aid of SFH spreading and MFSK modulation, the transmitted signal can be expressed as

$$
\begin{array}{r}
s(t)=\sqrt{2 P_{s} R_{c}} \sum_{n=-\infty}^{\infty} \sum_{i=-\infty}^{\infty} P_{T_{c}}\left(t-n T_{c}\right) P_{T_{s}}\left(t-i T_{s}\right) \\
\cdot \cos \left[2 \pi\left(f_{n}+f_{i}\right) t+\varphi_{n}+\varphi_{i}\right]
\end{array}
$$

where $P_{s}$ is the transmitted symbol's power without FEC, $R_{c}$ is the coding rate, $P_{T_{c}}(t)$ and $P_{T_{s}}(t)$ are unit pulses of duration $T_{c}$ and $T_{s}$, respectively, while $T_{c}$ and $T_{s}$ are the FH dwell interval and MFSK symbol duration, respectively. We assume that $L$ MFSK/RS symbols are transmitted in each FH dwell interval, i.e., $T_{c}=L T_{s}$. Furthermore, in Equation (1) $f_{n}$ is the hopping frequency during the $n$th hopping interval, $f_{i}$ is the $i$ th tone frequency associated with the $i$ th transmitted MFSK/RS data symbol. Finally, $\varphi_{n}$ and $\varphi_{i}$ are random phases during the $n$th FH interval and the $i$ th symbol interval.

The FH patterns are modeled as sequences of independent random variables, each of which is uniformly distributed over the set of legitimate FH frequencies. For each FH tone, the channel is modeled as a frequency non-selective fading process obeying Nakagami- $m$ distribution [5]. However, different FH tones encounter independent fading. Furthermore, the communication channel is assumed to be contaminated by both PBGI [6] and Additive White Gaussian Noise 
(AWGN). The PBGI occupies a fraction $\rho \leq 1$ of the band, and the power spectral density of this contaminating source is $N_{I} / 2 \rho$. The AWGN is included in the analysis for modeling the receiver's thermal noise, and it has a uniform spectral density of $N_{0} / 2$. Thus, in the portion of the frequency band contaminated by PBGI, the total two-sided power spectral density of the noise is $N_{n} / 2=N_{I} / 2 \rho+N_{0} / 2$. In the remainder of the band, the total noise power spectral density is $N_{n} / 2=N_{0} / 2$.

The demodulator consists of a bank of $M$ parallel noncoherent square-law detectors [6]. The detectors are followed by a decision device that employs a threshold test. The decision whether to erase a low-confidence symbol or not is made independently for each RS coded MFSK symbol. For those MFSK/RS symbols that are not erased, standard hard-decision demodulation is employed. The decision device is followed by an $M$-ary symbol deinterleaver and a decoder that employs EAE based RS decoding.

\section{STATISTICS OF THE ERASURE INSERTION RELATED VARIABLES}

In this section, various erasure insertion schemes using the OTT, RTT and the joint MO-RTT are investigated and the PDFs of the quantities involved in these erasure insertion schemes are derived. From these PDFs, we can gain an explicit insight into the properties of the related erasure insertion schemes. Furthermore, with the aid of these PDFs, the corresponding RS codeword decoding error probability using EAE decoding can be estimated using a numerical approach.

Let $H_{1}$ and $H_{0}$ represent the hypotheses of correct decisions and erroneous decisions, respectively, concerning an $M$-ary symbol in the MFSK demodulator using hard-detection. Furthermore, let $P_{N_{n}}\left(H_{1}\right)$ and $P_{N_{n}}\left(H_{0}\right)$ be the average correct and erroneous symbol probabilities, respectively, for a given value of $N_{n}$. Then, we have $P_{N_{n}}\left(H_{1}\right)=1-P_{N_{n}}\left(H_{0}\right)$, and $P_{N_{n}}\left(H_{0}\right)$ is given by

$$
\begin{gathered}
P_{N_{n}}\left(H_{0}\right)=1-\sum_{k=0}^{\infty} \sum_{n=0}^{M-1}(-1)^{k+n} \frac{(1-m)_{k}}{k !}\left(\begin{array}{c}
M-1 \\
n
\end{array}\right) \\
\cdot\left(\frac{m}{m+\gamma_{c}}\right)^{m}\left(\frac{\gamma_{c}}{m+\gamma_{c}}\right)^{-1}\left(\frac{\gamma_{c}}{m n+n \gamma_{c}+m}\right)^{k+1},
\end{gathered}
$$

where $m$ is the fading factor associated with the Nakagami- $m$ fading channels, $\gamma_{c}=R_{c} \Omega E_{s} / N_{n}=R_{c} \Omega b E_{b} / N_{n}, E_{s}=P_{s} T_{s}$ represents the transmitted energy per symbol without FEC, $b=\log _{2} M$ represents the number of bits per MFSK/RS symbol, and finally, we have $N_{n}=N_{0}+N_{I} / \rho$ with a probability of $\rho$, while $N_{n}=N_{0}$ holds with a probability of $(1-\rho)$.

Let $\left\{U_{1}, U_{2}, \ldots, U_{M}\right\}$ represent the decision variables input to the MFSK demodulator of Fig.1 in [2]. We denote the maximum and the 'second' maximum of $\left\{U_{1}, U_{2}, \ldots, U_{M}\right\}$ by

$$
\begin{aligned}
Y_{1} & =\max _{1}\left\{U_{1}, U_{2}, \ldots, U_{M}\right\}, \\
Y_{2} & =\max _{2}\left\{U_{1}, U_{2}, \ldots, U_{M}\right\} .
\end{aligned}
$$

In the context of the OTT, the decision variable subjected to an erasure insertion is $Y_{1}$, i.e., the actual demodulator output is observed. In order to analyze the properties of the OTT-based erasure insertion scheme and to estimate its corresponding EAE RS decoding performance, the PDFs $f_{Y_{1}}\left(y \mid H_{1}\right)$ and $f_{Y_{1}}\left(y \mid H_{0}\right)$, given that the associated demodulated symbol is correct $\left(H_{1}\right)$ and incorrect $\left(H_{0}\right)$, respectively, must be derived.
The ratio involved in Viterbi's RTT is defined as the ratio of the maximum to the 'second' maximum [3], or equivalently, it can be defined as the ratio of the 'second' maximum to the maximum, which can be expressed as $\lambda=Y_{2} / Y_{1}, 0 \leq \lambda \leq 1$. Similarly, in order to analyze the EAE RS decoding performance in terms of the RTT, the PDFs of $f_{\lambda}\left(r \mid H_{1}\right)$ and $f_{\lambda}\left(r \mid H_{0}\right)$, given that the associated demodulated symbol is correct $\left(H_{1}\right)$ and incorrect $\left(H_{0}\right)$ are required.

Finally, in the context of the joint MO-RTT, the erasure insertion is based on the observation of both the maximum $Y_{1}$ of (3) and the ratio $\lambda=Y_{2} / Y_{1}$. Hence, the joint two-dimensional (2D) PDFs of $f_{Y_{1}, \lambda}\left(y, r \mid H_{1}\right)$ and $f_{Y_{1}, \lambda}\left(y, r \mid H_{0}\right)$ are required, in order to estimate the EAE RS decoding performance in terms of the joint MO-RTT erasure insertion scheme. Note that, both $f_{Y_{1}}\left(y \mid H_{\vartheta}\right)$ and $f_{\lambda}\left(r \mid H_{\vartheta}\right)$ constitute the 'marginal' PDFs of the joint PDF of $f_{Y_{1}, \lambda}\left(y, r \mid H_{\vartheta}\right)$ associated with $\vartheta \in\{0,1\}$. Hence, their corresponding PDFs can be derived from the joint PDFs of $f_{Y_{1}, \lambda}\left(y, r \mid H_{\vartheta}\right)$ in terms of the MORTT.

The joint PDFs of $Y_{1}$ and $\lambda$ under the hypotheses $H_{1}$ of correction decision and $H_{0}$ of erroneous decision, respectively, can be expressed as

$$
\begin{gathered}
f_{Y_{1}, \lambda}\left(y, r \mid H_{1}\right)=\frac{(M-1) y}{P_{N_{n}}\left(H_{1}\right)}\left(\frac{m}{m+\gamma_{c}}\right)^{m} \\
\cdot \exp \left[-\left(\frac{m}{m+\gamma_{c}}+r\right) y\right][1-\exp (-y r)]^{M-2} \\
\cdot_{1} F_{1}\left(1-m, 1,-\frac{\gamma_{c} y}{m+\gamma_{c}}\right), 0 \leq y<\infty, 0 \leq r \leq 1, \\
f_{Y_{1}, \lambda}\left(y, r \mid H_{0}\right)=\frac{(M-1) y}{P_{N_{n}}\left(H_{0}\right)}\left\{\left(\frac{m}{m+\gamma_{c}}\right)^{m}\right. \\
\cdot \exp \left(-\frac{\left(m r+m+\gamma_{c}\right) y}{m+\gamma_{c}}\right)[1-\exp (-y r)]^{M-2} \\
\cdot{ }_{1} F_{1}\left(1-m, 1,-\frac{\gamma_{c} y r}{m+\gamma_{c}}\right) \\
+(m-2) \exp [-(r+1) y][1-\exp (-y r)]^{M-3} \\
\cdot[1-\Phi(y r)]\}, 0 \leq y<\infty, 0 \leq r \leq 1,
\end{gathered}
$$

where $P_{N_{n}}\left(H_{1}\right)=1-P_{N_{n}}\left(H_{0}\right)$, while $P_{N_{n}}\left(H_{0}\right)$ is given by (2), ${ }_{1} F_{1}()$ is the confluent hyper-geometric function, which is defined as ${ }_{1} F_{1}(a ; b ; x)=\sum_{k=0}^{\infty} \frac{(a)_{k} x^{k}}{(b)_{k} k !}, b \neq 0,-1,-2, \cdots$, where $(a)_{k}=$ $a(a+1)(a+2) \cdots(a+k-1),(a)_{0}=1$. Furthermore, in (6) we have

$$
\begin{aligned}
\Phi(y) & =\left(\frac{m}{m+\gamma_{c}}\right)^{m-1} \exp \left(-\frac{m y}{m+\gamma_{c}}\right) \\
& \times \sum_{k=0}^{\infty} \sum_{n=0}^{k} \frac{(1-m)_{k}}{k ! n !}\left(-\frac{\gamma_{c}}{m}\right)^{k}\left(\frac{m y}{m+\gamma_{c}}\right)^{n} .
\end{aligned}
$$

Upon integrating both sides of (5) and (6) in terms of the variable $\lambda$ from zero to one, we obtain the PDFs of $Y_{1}$ under the hypotheses $H_{1}$ of correction decision and $H_{0}$ of erroneous decision, which can 
be expressed as

$$
\begin{array}{r}
f_{Y_{1}}\left(y \mid H_{1}\right)=\frac{1}{P_{N_{n}}\left(H_{1}\right)}\left(\frac{m}{m+\gamma_{c}}\right)^{m} \exp \left(-\frac{m y}{m+\gamma_{c}}\right) \\
\cdot[1-\exp (-y)]^{M-1}{ }_{1} F_{1}\left(1-m, 1,-\frac{\gamma_{c} y}{m+\gamma_{c}}\right), \\
0 \leq y<\infty, \\
f_{Y_{1}}\left(y \mid H_{0}\right)=\frac{M-1}{P_{N_{n}}\left(H_{0}\right)} \exp (-y)[1-\Phi(y)] \\
\cdot[1-\exp (-y)]^{M-2}, 0 \leq y<\infty .
\end{array}
$$

Similarly, upon integrating both sides of (5) and (6) in terms of the variable $y$ from zero to infinity, we arrive at the PDFs of $\lambda$ under the hypotheses $H_{1}$ of correction decision and $H_{0}$ of erroneous decision, which can be expressed as

$$
\begin{array}{r}
f_{\lambda}\left(r \mid H_{1}\right)=\frac{M-1}{P_{N_{n}}\left(H_{1}\right)}\left(\frac{m}{m+\gamma_{c}}\right)^{m} \sum_{k=0}^{\infty} \sum_{n=0}^{M-2}(-1)^{n+k} \\
\cdot\left(\begin{array}{c}
M-2 \\
n
\end{array}\right) \frac{(k+1)(1-m)_{k}}{k !}\left(\frac{\gamma_{c}}{m+\gamma_{c}}\right)^{k} \\
\cdot\left(\frac{m+\gamma_{c}}{m+\left(m+\gamma_{c}\right)(n+1) r}\right)^{k+2}, 0 \leq r \leq 1, \\
f_{\lambda}\left(r \mid H_{0}\right)=\frac{M-1}{P_{N_{n}}\left(H_{0}\right)}\left\{\begin{array}{l}
\sum_{k=0}^{\infty} \frac{(1-m)_{k}}{k !}\left(\frac{m}{m+\gamma_{c}}\right)^{m-3} \\
\cdot\left[\left(\frac{m}{\gamma_{c} r}\right)^{2}\left(\frac{m}{m+\gamma_{c}}\right)^{M-2}(-1)^{n+k}\left(\begin{array}{c}
M-2 \\
n
\end{array}\right)\right. \\
\cdot(k+1)\left(\frac{\gamma_{c} r}{\left(m n+n \gamma_{c}+m\right) r+m+\gamma_{c}}\right)^{k+2} \\
-(M-2) \sum_{j=0}^{k} \sum_{n=0}^{M-3}(-1)^{n+k}(j+1)\left(\frac{\gamma_{c}}{m}\right)^{k}\left(\begin{array}{c}
M-3 \\
n
\end{array}\right) \\
\cdot\left(\frac{m}{(n+1)\left(m+\gamma_{c}\right) r+2 m+\gamma_{c}}\right)^{j+2}
\end{array}\right] \\
+(M-2) \sum_{n=0}^{M-3}(-1)^{n}\left(\begin{array}{c}
M-3 \\
n
\end{array}\right)^{2}\left(\frac{1}{n r+r+1}\right\},
\end{array}
$$

The properties of the proposed OTT, Viterbi's RTT as well as MO-RTT can be studied with the aid of their corresponding PDFs $f_{Y_{1}}\left(y \mid H_{1}\right)$ and $f_{Y_{1}}\left(y \mid H_{0}\right)$ for the OTT shown in Fig.1, using $f_{\lambda}\left(r \mid H_{1}\right)$ and $f_{\lambda}\left(r \mid H_{0}\right)$ for the RTT in Fig.2, while invoking the joint 2D PDFs of $f_{Y_{1}, \lambda}\left(y, r \mid H_{1}\right)$ and $f_{Y_{1}, \lambda}\left(y, r \mid H_{0}\right)$ for the MO-RTT in Fig.3 for a range of parameters, which are summarized in the figures ${ }^{1}$. In the context of the OTT of Fig.1, we observe that $f_{Y_{1}}\left(y \mid H_{1}\right)$ is mainly distributed over a normalized demodulator output range associated with relatively high values of $Y_{1}$, while $f_{Y_{1}}\left(y \mid H_{0}\right)$ is spread over a range having relatively low values of $Y_{1}$. Consequently, we can argue that the demodulated symbols having relatively low values of $Y_{1}$ are less reliable than those having relatively high values of $Y_{1}$. Let $Y_{T}$ be a threshold associated with making an erasure decision based on the

\footnotetext{
${ }^{1}$ More figures in the context of the PDFs of the OTT, RTT and MO-RTT can be found at http://wwwmobile.ecs.soton.ac.uk/lly/html_file/errors_and_erasures.html
}

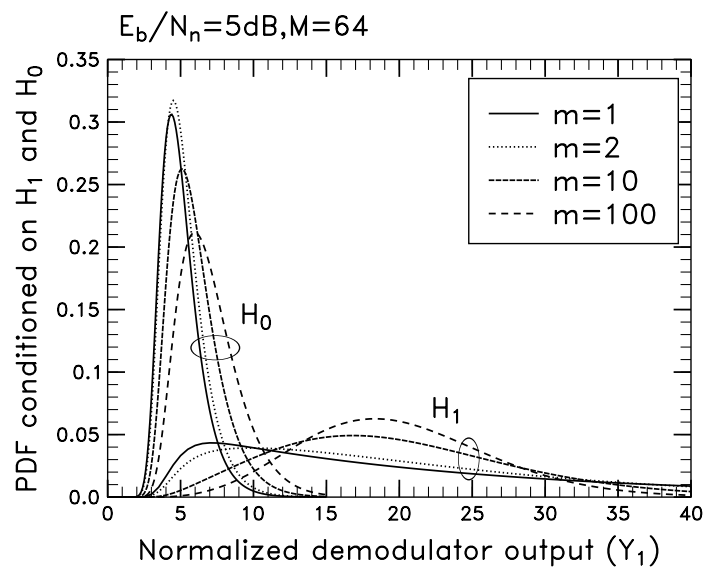

Figure 1: The PDFs of $Y_{1}=\max \{\cdot\}$ under the hypotheses of $H_{1}$ and $H_{0}$ using $M=64$, average SINR per bit of $E_{b} / N_{n}=5 \mathrm{~dB}$ and Nakagami fading parameters of $m=1,2,10,1000$ over flat Nakagami- $m$ fading channels.

OTT. Then, if $Y_{1} \leq Y_{T}$, the associated demodulated symbol should be erased. Otherwise, if $Y_{1}>Y_{T}$, the demodulator outputs a RS code symbol. In the context of the RTT of Fig.2, we observe that $f_{\lambda}\left(r \mid H_{1}\right)$ is mainly spread over the range having relatively low values of $\lambda$, while $f_{\lambda}\left(r \mid H_{0}\right)$ is mainly distributed over the range having relatively high values of $\lambda$. Therefore, the demodulated symbols having a relatively low ratio of $\lambda$ are more reliable, than those having relatively high values of $\lambda$. Consequently, a pre-set threshold $\lambda_{T}$ can be invoked, in order to erase these low-reliability symbols associated with a ratio of $\lambda \geq \lambda_{T}$. Finally, in the context of the joint MO-RTT having the PDFs seen in Fig.3, we observe that for the given parameters the peak of the distribution $f_{Y_{1}, \lambda}\left(y, r \mid H_{1}\right)$ is located at a relatively high value of $Y_{1}$ or a relatively low value of $\lambda$, while the peak of the distribution $f_{Y_{1}, \lambda}\left(y, r \mid H_{0}\right)$ is located at a relatively low value of $Y_{1}$ and a relatively high value of $\lambda$. The above observations in turn imply that $f_{Y_{1}, \lambda}\left(y, r \mid H_{1}\right)$ is mainly spread over the range having relatively high values of $Y_{1}$ or relatively low values of $\lambda$, while $f_{Y_{1}, \lambda}\left(y, r \mid H_{0}\right)$ is mainly distributed over the range having relatively low values of $Y_{1}$ and relatively high values of $\lambda$. Therefore, if a demodulated symbol has a maximum output value of $Y_{1}$ and a ratio of $\lambda$, that had fallen in the main range of $f_{Y_{1}, \lambda}\left(y, r \mid H_{0}\right)$, the symbol concerned must be a low-reliability symbol and must be replaced by an erasure. By contrast, if a demodulated symbol has values of $Y_{1}$ and $\lambda$ that had fallen outside the main range of $f_{Y_{1}, \lambda}\left(y, r \mid H_{0}\right)$, then this symbol is likely to be correct and the corresponding RS code symbol can be forwarded to the RS decoder. Consequently, in order to erase the low-reliability RS coded symbols, we assume that $Y_{T}$ and $\lambda_{T}$ are two thresholds, which activate an erasure insertion, whenever $Y_{1} \leq Y_{T}$ and $\lambda \geq \lambda_{T}$.

\section{NUMERICAL RESULTS OF CODEWORD DECODING ERROR PROBABILITY}

In the previous subsection we have given the required PDFs for the OTT, RTT and MO-RTT erasure insertion schemes. With the aid of these PDFs, the codeword decoding error probabilities using EAE RS 


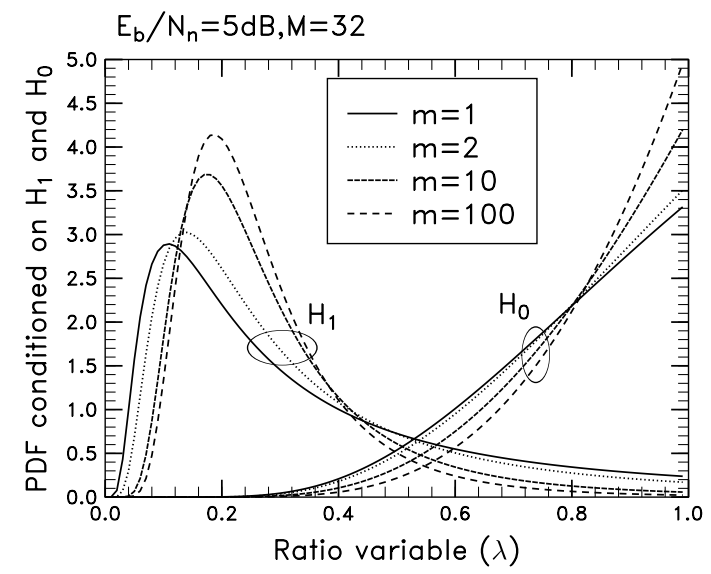

Figure 2: The PDFs of $\lambda=Y_{2} / Y_{1}$ under the hypotheses of $H_{1}$ and $H_{0}$ using $M=32$, an average SINR per bit of $E_{b} / N_{n}=5 \mathrm{~dB}$ and Nakagami fading parameters of $m=1,2,10,1000$.

decoding in terms of these erasure insertion schemes can now be estimated using numerical approaches. In this section the performance of the RS coded MFSK based SFH/SS system using EAE decoding associated with the OTT, RTT or MO-RTT erasure insertion scheme is estimated and compared for a range of parameters.

In Fig. 4 we investigated the influence of the PBGI duty factor, $\rho$, on the average BER performance of an uncoded MFSK based SFH/SS system over Nakagami- $m$ fading channels for fading factors of $m=$ $1,2,3,4,5,10$ and 100 . The parameters used were $M=32$, signal to noise ratio (SNR) per bit of $E_{b} / N_{0}=10 \mathrm{~dB}$ and signal to interference ratio (SIR) per bit of $E_{b} / N_{I}=8 \mathrm{~dB}$. From the results of Fig. 4 we observe that for each curve associated with a fading factor of $m>1$, there exists a PBGI duty factor, $\rho$, which results in the highest BER. By contrast, for the Rayleigh fading channel corresponding to $m=1$, the maximum BER is observed, when $\rho=1$, which implies that spreading the PBGI power over the whole band used results in the worst possible BER.

Fig. 5 shows the codeword decoding error probability of the RS coded SFH/SS system over the Nakagami- $m$ fading channel having $m=2$ using the proposed joint MO-RTT. The RS $(32,20)$ code over the Galois Field $\mathrm{GF}(32)=\mathrm{GF}\left(2^{5}\right)$ corresponding to 5 -bit symbols was used and EAE decoding based on the proposed MO-RTT insertion scheme was employed. From the results we observe that there exists an optimum threshold value of $\lambda_{T}$ or $Y_{T}$, for which the EAE decoding achieves the minimum codeword decoding error probability. This observation in turn implies that for given values of $M$, SNR per bit of $E_{b} / N_{0}$, SIR per bit of $E_{b} / N_{I}$ as well as for a given RS code, there exist optimum thresholds of $Y_{T}$ and $\lambda_{T}$, for which the EAE decoding using the joint MO-RTT erasure insertion scheme achieves the minimum codeword decoding error probability. This minimum codeword decoding error probability is lower, than that associated with using the RTT alone or the OTT alone. Note that the point corresponding to $Y_{T}=0$ and $\lambda_{T}=1$ represents the codeword decoding error probability using ECO decoding, i.e., no erasure insertion at all. Therefore, we can observe that the EAE decoding outperforms the ECO decoding, if the appropriate thresholds are invoked. However,

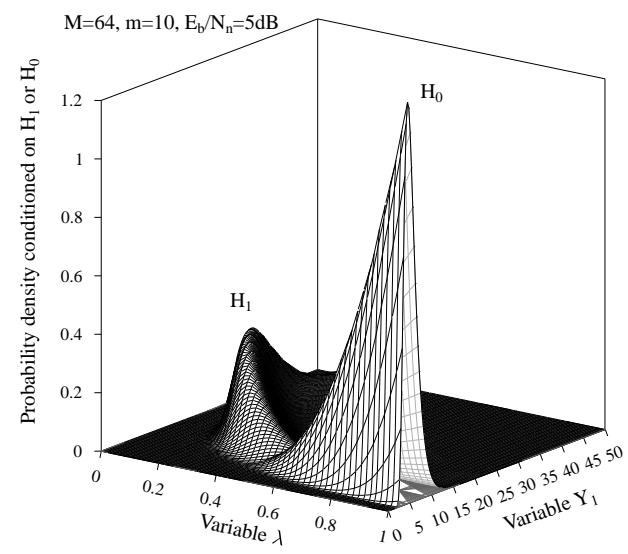

Figure 3: The joint 2D PDFs of $f_{Y_{1}, \lambda}\left(y, r \mid H_{1}\right)$ and $f_{Y_{1}, \lambda}\left(y, r \mid H_{0}\right)$ using parameters of $M=64, m=10$ and an average SINR per bit of $E_{b} / N_{n}=5 \mathrm{~dB}$ over flat Nakagami- $m$ fading channels.

if the threshold $Y_{T}$ is too high and simultaneously the threshold $\lambda_{T}$ is too low, too many erasures will be activated, potentially erasing correct demodulated symbols. Consequently, the codeword decoding error probability using EAE decoding might be higher, than that using ECO decoding.

Finally, in Fig.6 we evaluated the codeword decoding error probability of a RS $(32,20)$ coded MFSK based SFH/SS system employing the OTT, RTT and MO-RTT erasure insertion schemes over Nakagami$m$ fading channels having different fading parameters. In these investigations, we assumed that the optimum thresholds were employed, whenever EAE decoding was used. From the results we observe that both the proposed OTT and MO-RTT erasure schemes outperform the RTT erasure insertion scheme, and as predicted, the MO-RTT erasure scheme outperforms both the RTT and OTT erasure insertion schemes, provided that all the erasure insertion schemes are operated at the optimum decision thresholds. However, when the channel quality is poor $(m=1,2)$ or the SIR per bit is low $\left(E_{b} / N_{I}<9 \mathrm{~dB}\right)$, the codeword decoding error probability of the OTT and MO-RTT becomes similar.

\section{REFERENCES}

[1] M. B. Pursley and W. E. Stark, "Performance of ReedSolomon coded frequency-hop spread-spectrum communications in partial-band interference," IEEE Transactions on Communications, vol. 33, pp. 767-774, August 1985.

[2] Y. T. Su and L. der Jeng, "Antijam capability analysis of RScoded slow frequency-hopped system," IEEE Transactions on Communications, vol. 48, pp. 270-281, February 2000.

[3] A. J. Viterbi, "A robust ratio-threshold technique to mitigate tone and partial band jamming in coded MFSK systems," in Proceedings of IEEE Military Communications Conferences Rec., pp. 22.4.1-22.4.5, IEEE, October 1982.

[4] C. W. Baum and M. B. Pursley, "Bayesian methods for erasure insertion in frequency-hop communication system with partial- 


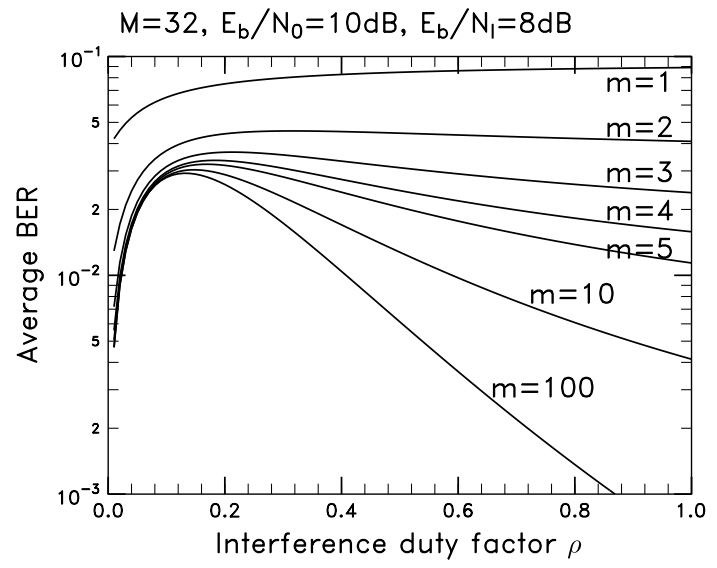

Figure 4: Average BER versus PBGI duty factor, $\rho$, performance for the uncoded MSFK based SFH/SS system over Nakagami- $m$ fading channels with PBGI.

band interference," IEEE Transactions on Communications, vol. 40, pp. 1231-1238, July 1992.

[5] M. K. Simon and M.-S. Alouini, "A unified approach to the performance analysis of digital communication over generalized fading channels," Proceedings of the IEEE, vol. 86, pp. 1860-1877, September 1998

[6] R. E. Ziemer and R. L. Peterson, Digital Communications and Spread Spectrum Systems. New York: Macmillan Publishing Company, 1985.

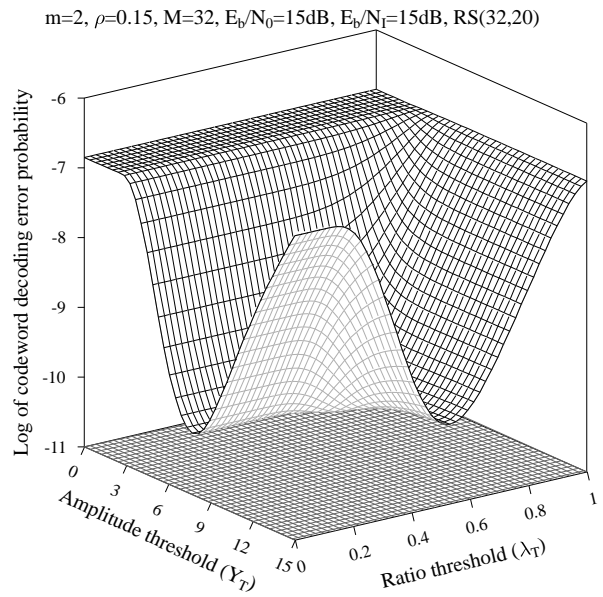

Figure 5: Codeword decoding error probability versus the amplitude threshold, $Y_{T}$ and the ratio threshold, $\lambda_{T}$ for the RS(32,20) FEC code using EAE decoding based on the MO-RTT erasure insertion scheme over Nakagami- $m$ fading channels with PBGI.

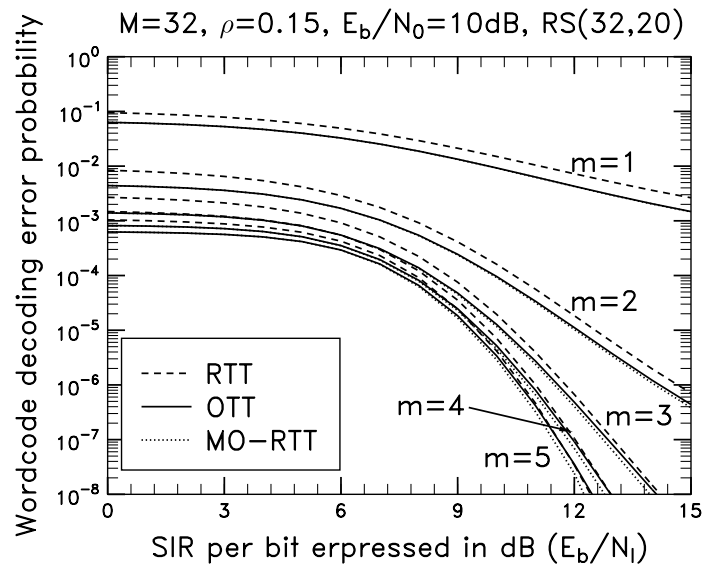

Figure 6: Codeword decoding error probability versus signal to interference ratio (SIR) per bit for RS $(32,20)$ EAE decoding using the OTT, RTT and joint MO-RTT erasure insertion schemes over the Nakagami- $m$ fading channels with PBGI. 